\title{
Sex and adverse events of adjuvant chemotherapy in colon cancer: an analysis of 34,640 patients in the ACCENT database
}

Anna D. Wagner, M.D. ${ }^{1}$; Axel Grothey, M.D. ${ }^{2}$; Thierry Andre, M.D. ${ }^{3}$; Jesse G. Dixon, M.S. ${ }^{4}$; Norman Wolmark, M.D"5; Daniel G. Haller, M.D. ${ }^{6}$; Carmen J. Allegra, M.D.7; Aimery de Gramont, M.D. ; Eric VanCutsem, M.D. Ph.D. ${ }^{9}$; Steven R. Alberts, M.D. ${ }^{10}$; Thomas J. George, M.D. ${ }^{11}$; Michael J. O'Connell, M.D. ${ }^{12}$; Christopher Twelves, M.D. ${ }^{13}$; Julien Taieb, M.D. Ph.D. ${ }^{14}$; Leonard B. Saltz, M.D. ${ }^{15}$; Charles D. Blanke, M.D ${ }^{16}$; Edoardo Francini, M.D. Ph.D. ${ }^{17}$; Rachel Kerr, M.D. ${ }^{18}$; Greg Yothers, M.D. Ph.D. ${ }^{19}$; Jean F. Seitz M.D. ${ }^{20}$; Silvia Marsoni, M.D. ${ }^{21}$; Richard M. Goldberg, M.D. ${ }^{22}$; Qian Shi, Ph.D. ${ }^{4}$

${ }^{1}$ Department of Oncology, Division of Medical Oncology, Lausanne University Hospital and University of Lausanne, Lausanne, Switzerland; ${ }^{2}$ West Cancer Center and Research Institute, Germantown, TN, USA; ${ }^{3}$ Sorbonne University and Saint-Antoine Hospital, Paris, France; ${ }^{4}$ Division of Biomedical Statistics and Informatics, Mayo Clinic, Rochester, NY, USA; ${ }^{5}$ RRG Oncology and the University of Pittsburgh, Pittsburgh, PA, US; ${ }^{6}$ Abramson Cancer Center, Philadelphia, USA; ${ }^{7}$ The University of Florida, Gainesville, FL, USA; ${ }^{8}$ Franco-British Institute, Levallois-Perret, France; ${ }^{9}$ University of Leuven, Leuven, Belgium; ${ }^{10}$ Department of Oncology, Mayo Clinic, Rochester, NY, USA; ${ }^{11}$ Department of Medicine and University of Florida Health Cancer Center, Gainesville, USA; ${ }^{12}$ NSABP Foundation, Pittsburgh, PA, USA; ${ }^{13}$ Leeds Teaching

(C) The Author(s) 2020. Published by Oxford University Press. All rights reserved. For permissions, please email: journals.permissions@oup.com 
Hospitals Trust and University of Leeds, Leeds, UK; ${ }^{14}$ Department of Gastroenterology, GeorgesPompidou European Hospital, AP-HP, Sorbonne Paris Cité, Université de Paris, Paris, France;

${ }^{15}$ Memorial Sloan Kettering Cancer Center, New York, USA; ${ }^{16}$ Southwest Oncology Group, Ann Arbor, MI, USA; ${ }^{17}$ University of Florence, Florence, Italy; ${ }^{18}$ Adjuvant Colorectal Cancer Group, University of Oxford, UK; ${ }^{19}$ NRG Oncology and the University of Pittsburgh; ${ }^{20}$ Timone Hospital, Aix-Marseille-University, Marseille, France; ${ }^{21}$ Precision Oncology, The FIRC Institute Of Molecular Oncology, Milan, Italy; ${ }^{22}$ West Virginia University Cancer Institute, Morgantown, USA

\section{Corresponding author:}

Dr. Anna D. Wagner, Department of Oncology, Division of Medical Oncology, Lausanne University Hospital and University of Lausanne, Bugnon 46, Lausanne, 1011, Switzerland, CH Phone: +41 7955 61889,

Email: dorothea.wagner@chuv.ch 


\section{Abstract}

Background: Adjuvant chemotherapy is a standard treatment option for patients with stage III and high-risk stage II colon cancer. Sex is one of several factors responsible for the wide interpatient variability in drug responses. Amalgamated data on the effect of sex on the toxicity of current standard adjuvant treatment for colorectal cancer are missing.

Methods: Objective of our study was to compare incidence and severity of major toxicities of fluoropyrimidine- (5-FU or capecitabine) based adjuvant chemotherapy, with or without oxaliplatin, between male and female patients after curative surgery for colon cancer. Adult patients enrolled in 27 relevant randomized trials included in the ACCENT database, a large, multi-group, international data repository containing individual patient data, were included. Comparisons were conducted using logistic regression models (stratified by study and treatment arm) within each type of adjuvant chemotherapy (5FU, FOLFOX, Capecitabine, CAPOX, and FOLFIRI). The following major toxicities were compared (grade III/IV and grade I-IV, according to $\mathrm{NCl}-\mathrm{CTC}$ criteria, regardless of attribution): nausea, vomiting, nausea/vomiting, stomatitis, diarrhea, leukopenia, neutropenia, thrombocytopenia, anemia, and neuropathy (in patients treated with oxaliplatin).

Results: Data from 34,640 patients were analyzed. Statistically significant and clinically relevant differences in the occurrence of grade III/IV non-hematological (especially nausea [5FU: Odds Ratio $(\mathrm{OR})=2.33,95 \%$ Confidence Interval $(\mathrm{Cl})=1.90$ to 2.87 , $\mathrm{p}$-value $<.001$, FOLFOX: OR $=2.34$, $95 \% \mathrm{Cl}=1.76$ to $3.11, \mathrm{p}$-value $<.001$ ], vomiting [5FU: $\mathrm{OR}=2.38,95 \% \mathrm{Cl}=1.86$ to $3.04, \mathrm{p}$-value $<.001$, FOLFOX: OR $=2.00,95 \% \mathrm{Cl}=1.50$ to 2.66 , $\mathrm{p}$-value $<.001, \mathrm{CAPOX}: \mathrm{OR}=2.32,95 \% \mathrm{Cl}=1.55$ 
to $3.46, \mathrm{p}$-value <.001], and diarrhea [5FU: $\mathrm{OR}=1.35,95 \% \mathrm{Cl}=1.21$ to $1.51, \mathrm{p}$-value $<.001$, FOLFOX: $O R=1.60,95 \% \mathrm{Cl}=1.35$ to 1.90 , $\mathrm{p}$-value $<.001$, FOLFIRI: $\mathrm{OR}=1.57,95 \% \mathrm{Cl}=1.25$ to 1.97 , $\mathrm{p}$-value $<.001$ ), as well as hematological toxicities (neutropenia [5FU: $\mathrm{OR}=1.55,95 \% \mathrm{Cl}=1.37$ to 1.76, $\mathrm{p}$-value <.001, FOLFOX: $\mathrm{OR}=1.96,95 \% \mathrm{Cl}=1.71$ to $2.25, \mathrm{p}$-value <.001, FOLFIRI: OR $=2.01$, $95 \% \mathrm{Cl}=1.66$ to $2.43, \mathrm{p}$-value $<.001$, Capecitabine: $\mathrm{OR}=4.07,95 \% \mathrm{Cl}=1.84$ to $8.99, \mathrm{p}$-value <.001] and leukopenia [5FU: $\mathrm{OR}=1.74,95 \% \mathrm{Cl}=1.40$ to 2.17 , $\mathrm{p}$-value <.001, FOLFIRI: OR =1.75, $95 \% \mathrm{Cl}=1.28$ to $2.40, \mathrm{p}$-value $<.001]$ ), were observed, with women being consistently at increased risk.

Conclusions: Our analysis confirms that women with colon cancer receiving adjuvant fluoropyrimidine-based chemotherapy are at increased risk of toxicity. Given the known sex differences in fluoropyrimidine pharmacokinetics, sex-specific dosing of fluoropyrimidines warrants further investigation. 
An individuals' sex is one of the most important modulators of disease risk and response to treatment [1]. The importance and potential for sex and gender analyses to foster scientific discovery has been highlighted recently [2]. A growing number of peer-reviewed journals now require sex- or gender-specific reporting $[3,4]$. In fact, the Journal of the National Cancer Institute was the first journal to include instructions for addressing the effects of sex as part of its manuscript preparation policy [5]. Although ESMO recently addressed the topic [6], oncology lags behind other disciplines, such as cardiology. A PubMed search for "sex", "toxicity", and "chemotherapy", conducted in 2016 before initiating this analysis, including all solid tumors, identified 11 studies including more than 100 participants. Available studies in patients with colorectal cancer did not include prospective data on currently used oxaliplatinor capecitabine-based regimens.

Adjuvant chemotherapy is standard for patients with stage III and high-risk stage II colon cancer. The impact of a patient's sex on the incidence and severity of adverse events has not, however, been well documented. Of note, while self-reported gender is that what is being reported, given the low frequency of transgender persons (0.3-0.5\%) [7], we can assume biological sex and gender to be identical in 99.5 and $99.7 \%$ of patients. Therefore, as we consider biological differences between men and women as primarily responsible for potential differences in treatment effects, throughout this manuscript, we will use the term sex differences, and refer to males and females.

This study aimed to compare between the sexes the incidence and severity of major adverse events of clinically relevant fluoropyrimidine (5-FU or capecitabine)-based adjuvant chemotherapy, with or without oxaliplatin or irinotecan after curative surgery for colon cancer 
in a large population of clinical trial participants who are part of the ACCENT database.

\section{Methods}

\section{Trial Selection}

This is a secondary analysis of previously conducted trials and was approved by Mayo Clinic Institutional Review Board. Patients provided informed consent for participating in the original trials, which were obtained by local enrolling centers.

All adult patients with colorectal cancer who participated in any of the 27 relevant randomized clinical trials of adjuvant chemotherapy that comprise the ACCENT database were included. A list of all trials and treatment arms included in this analysis is provided as supplementary material (supplementary table 1). ACCENT (Adjuvant Colon Cancer End Points) $[8,9]$ is a large, multi-group, international data repository containing individual patient level information from clinical trials. Categories of chemotherapy regimens were 5-FU single-agent (plus folinic acid), with or without oxaliplatin (e.g., FOLFOX, FLOX), Capecitabine as a singleagent or in combination with oxaliplatin (CAPOX) and 5-FU (plus folinic acid) plus irinotecan (FOLFIRI and other regimens).

Although FOLFIRI is not a standard adjuvant treatment, but given its frequent use in patients with metastatic disease, trials including this chemotherapy combination were also analyzed. Patients assigned to combinations of chemotherapy plus targeted treatments no longer used as adjuvant treatment, such as FOLFOX plus bevacizumab, FOLFOX plus cetuximab, or CAPOX plus bevacizumab, representing 14 treatment arms from 13 trials, were excluded. 


\section{Statistical Analysis}

We analyzed patient characteristics and adverse events separately for each regimen category. Chi-square test was used to detect differences in baseline characteristics between male and female patients. The following major adverse events were compared (grade III/IV and grade I-IV, according to $\mathrm{NCl}$-criteria, regardless of attribution) between males and females: nausea, vomiting, nausea/vomiting, stomatitis, diarrhea, leukopenia, neutropenia, thrombocytopenia, anemia, and neuropathy, in patients treated with oxaliplatin. Results for comparisons between grade I-IV adverse events are included in the supplementary materials. Total patients included in logistic models for each AE differed due to data availability including specific AE data missing per patient and specific AE data missing per study. In order to assess the association between AEs and sex, odds ratios were calculated using multivariate logistic models adjusting for age, grade, stage, performance score (PS), and body mass index (BMI) and compared using stratified Wald test. The interaction effects between sex and adjusting variables were tested using stratified Wald test and none of them were found to be statistically significant. In order to account for study and treatment-specific differences, logistic models were stratified by study and treatment arm. Two-sided p-values are reported. We designated comparisons in which the $p$-value was <.001 as statistically significant to adjust for multiple comparisons.

\section{Results}

This analysis included 34,640 patients with a median age for both males and females of 61.0 years. Clinical characteristics with statistically significant $(p<.001)$ differences are 
displayed in Table 1 according to chemotherapy regimen. Across chemotherapy regimens, we noted statistically significant differences in patients' characteristics between the sexes.

Specifically, females had more often a BMI of $<18.5$ (4.5\% females versus $1.2 \%$ males) or 18.5 $25 \mathrm{~kg} / \mathrm{m}^{2}$ (46.3\% females versus $39.4 \%$ males), whereas males more often had a $\mathrm{BMI}$ of $\geq 25$ $\mathrm{kg} / \mathrm{m}^{2}$ (59.3\% males versus $49.2 \%$ females); females were more often under 50 years old (18.9\% females versus $16.8 \%$ males), whereas males more often were $\geq 65$ years old $(37.5 \%$ males versus $36.3 \%$ females); and females more often had a performance score of 1 (20.8\% females versus $18.6 \%$ males), whereas males more often had a performance score of 0 ( $80.5 \%$ males versus $78.5 \%$ females).

While the differences in BMI are clearly considered as clinically meaningful, differences in age groups and PS are less important. Overall, 17 treatment-related deaths were observed, 12 females and five males (Chi-square p-value=.04. Due to the rarity of these events, we excluded them from the logistic models, which were limited to grade I-IV and grade III/IV adverse events. The adjusted associations between grade III/IV hematologic AEs and sex according to treatment regimen are displayed in Figure 1. While the odds of experiencing grade III/IV thrombocytopenia and anemia were comparable, female patients had higher odds of experiencing grade III/IV neutropenia (5FU: OR 1.55, 95\% Cl 1.37-1.76, p-value <.001, FOLFOX: OR 1.96, 95\% Cl 1.71-2.25, p-value <.001, FOLFIRI: OR 2.01, 95\% Cl 1.66-2.43, p-value <.001, Capecitabine: OR 4.07, 95\% Cl 1.84-8.99, p-value <.001) and leukopenia (5FU: OR 1.74, 95\% Cl 1.40-2.17, p-value <.001, FOLFIRI: OR 1.75, 95\% Cl 1.28-2.40, p-value <.001) which reached statistical significance within at least one treatment subgroup. The adjusted associations between grade III/IV non-hematologic adverse events are shown in Figure 2. Again, 
female patients had higher odds of experiencing grade III/IV nausea (5FU: OR $2.33,95 \% \mathrm{Cl} 1.90$ 2.87, p-value <.001, FOLFOX: OR 2.34, 95\% Cl 1.76-3.11, p-value <.001), vomiting (5FU: OR 2.38, 95\% Cl 1.86-3.04, p-value <.001, FOLFOX: OR 2.00, 95\% Cl 1.50-2.66, p-value <.001, CAPOX: OR 2.32, $95 \% \mathrm{Cl}$ 1.55-3.46, $\mathrm{p}$-value <.001), stomatitis (5FU: OR 2.20, $95 \% \mathrm{Cl} 1.82-2.66, \mathrm{p}$-value <.001), diarrhea (5FU: OR 1.35, 95\% Cl 1.21-1.51, p-value <.001, FOLFOX: OR 1.60, 95\% Cl 1.351.90, p-value <.001, FOLFIRI: OR 1.57, $95 \% \mathrm{Cl} 1.25-1.97, \mathrm{p}$-value <.001), peripheral neuropathy (FOLFOX: OR 1.34, 95\% Cl 1.15-1.57, p-value <.001), and transaminitis (FOLFOX: OR 2.45, 95\% Cl 151-3.96, $p$-value $<.001)$ which reached statistical significance within at least one treatment subgroup, while the odds of experiencing grade III/IV peripheral neuropathy (only CAPOX subgroup), rash, handfoot syndrome, and transaminitis (with the exception of FOLFOX subgroup) were comparable. Adjusted associations between grade I-IV hematological and nonhematological adverse events according to sex and treatment regimen are available as supplementary material.

\section{Discussion}

Including 34,640 patients, our analysis is the largest to date to address in a systematic manner the impact of a patients' sex on the toxicity of all currently used adjuvant chemotherapy regimens. Importantly, for the first time we report prospectively collected data on oxaliplatin-based and capecitabine-based regimens. We confirm that female patients with colon cancer consistently experience clinically and statistically significant greater toxicity. This effect is seen across regimens and most adverse events, but is greatest for severe neutropenia and leukopenia. 
Variability in outcomes in either efficacy or toxicity can broadly be broken down into two categories. Pharmacokinetic variability reflects differences within populations with respect to the extent of drug exposure due, for example to differences in absorption or metabolism. By contrast, pharmacodynamic variability is the result of differences in the biological effects of a drug between patients with the same drug exposure. A patient's sex is known to affect both the pharmacokinetics of drug disposition and the pharmacodynamics of drug sensitivity [10] but is usually not taken into account for dosage individualization. In addition, current chemotherapy dosing according to body surface area neither takes into account the sex differences in fat-free body-mass [6], nor the large individual differences in body composition among patients with a similar body surface area.

Our findings raise several important questions. The first is: how can we explain the observed differences in toxicity, and what are the roles of genetic and non-genetic factors? While the patients' sex has no effect on the clearance of oxaliplatin [11], sex differences in the clearance of 5-FU [12, 13], which are independent of age [14], are likely to explain the differences in toxicity observed. As a consequence of their lower clearance of 5-FU, dosing according to body surface area results in higher plasma fluoropyrimidine levels in females [1214].

Although the precise reason for the lower clearance of 5-FU in females is not certain, the major route of elimination of 5-FU is hepatic metabolism by the enzyme dihydropyrimidine dehydrogenase (DPYD). DPYD activity is associated with fluoropyrimidine toxicity [15], but data on sex differences in DPYD activity are controversial [16-18]. A strong interaction between DPYD genetics and sex has been observed by different authors [18-20], with a greater 
predictive impact of several DPD variants in males. By contrast, the lower clearance of 5-FU explains the higher toxicity in females. These observations provide a strong case for a sexspecific approach to personalized fluoropyrimidine dosing. Sex differences in body composition, including the higher percentage of metabolically active, fat-free body mass in men [6] may also be relevant, because 5-FU pharmacokinetics are better predicted by fat-free mass and total body weight than standard anthropometric parameters [21].

The second major question raised by our analysis is whether the higher plasma levels and toxicity in females translate in a higher treatment efficacy. In this context, a recently presented, pooled analysis including 18.399 patients in $1^{\text {st }}$ line chemotherapy trials for metastatic colorectal cancer [22] confirmed the higher toxicity, but demonstrated equal efficacy of chemotherapy in females and males in terms of both progression-free- and overall survival. Thus, differences in pharmacodynamics must be postulated. Whether the tolerability of chemotherapies with greater toxicity in female patients could be improved by either dosereductions or intensification of supportive care measures only in female patients, and if dosereductions would decrease the efficacy are further important open questions.

The third major question is whether conventional dosing of 5-FU results in suboptimal therapeutic plasma levels in males? The overall lower frequency of toxicities in men could be interpreted as a sign of relative under-dosing. Body surface area -based dosing was applied to individualizing chemotherapy doses in the 1950s and has remained the default approach, although its inaccuracy, including the risk of under-dosing, has been recognized for more than 15 years [23]. Accordingly, pharmacologists have proposed adjusting doses up or down based upon a biologically relevant endpoint, such as myelosuppression [23, 24]. Moreover, in patients 
with metastatic colorectal cancer, an association between treatment with FOLFOX or trifluridine/tiperacil and improved median survival in patients with neutropenia (median survival in patients with grade III/IV neutropenia versus without neutropenia for FOLFOX 20.7 versus 12.5 months, $p<.001$; for trifluridine/tiperacil 9.8 versus 4.4 months) $[25,26]$ has been reported. A relatively small study of 32 participants confirms that conventional dosing of 5-FU results in "sub-therapeutic" plasma levels in the majority of males [13]. In a separate study of 152 patients, 124 were considered to have "sub-therapeutic" 5-FU levels [27]. To achieve "therapeutic" 5-FU levels, the mean 5-FU dose was higher in males (1,837 versus 1,763 $\mathrm{mg} / \mathrm{m}^{2} /$ week), respectively [27].

Arguably, a question of major importance is whether conventional dosing results in suboptimal treatment outcomes in males. A close relationship between plasma levels of 5-FU and toxicity/efficacy has been observed in patients with several tumor types (for review see [28]). A previous analysis of the ACCENT database showed that males had inferior time to recurrence (HR 1.05, 95\% Cl 1.01-1.09) and other efficacy endpoints after adjusting for age, stage, and treatment [8]. Interestingly, the stage of disease and type of adjuvant regimen did not influence the prognostic value of sex [8]. Another earlier ACCENT database analysis also showed that male sex, along with other patient and disease characteristics, was associated with increased early (<6 months) mortality [9].

A key strength of our analysis is the large number of patients included, which allows the identification of sex differences with clinical relevance and statistical significance. Furthermore, it enables to understand their magnitude while avoiding the risk of errors due to multiple testing observed in smaller datasets. Additional strong points are the inclusion of all currently 
relevant chemotherapy regimens, in which patients do not have confounding factors such as prior chemotherapy, which might complicate the interpretation of apparent differences in toxicity. Our analysis is, however, limited by the fact that not all types of toxicity were included in the ACCENT database. For example, data on neutropenic fever, lethargy, or fatigue are absent. Interestingly, a recently published analysis of the phase III PETACC-3 trial of FOLFIRI observed all-grade lethargy in $48.9 \%$ of females, as compared to $38.2 \%$ of males $(p<.001)$ [29]. Furthermore, trials usually report the worst grade of toxicity and not how many times it occurred in an individual patient. By necessity, therefore, we focus on differences in incidence rather than frequency of a given toxicity; likewise, the durations of these toxicities are also unavailable. Finally, data on dose reductions and delays, serious adverse events, and hospitalizations due to toxicity, are not captured in the ACCENT database.

In conclusion, the current analysis raises several important questions, including whether males should receive higher doses of 5-FU and whether this may increase the effectiveness of adjuvant chemotherapy in males with colon cancer, and whether females should receive either reduced doses of 5-FU or different and more intensive supportive treatments. Previous trials including pharmacokinetically-adjusted dosing $[27,30]$ confirmed that the balance between efficacy and toxicity of fluoropyrimidines may be improved statistically significantly and clinically relevant, but did not change clinical practice. Therefore, further rationally-designed, prospective clinical trials investigating alternatives to body surface area-based dosing of fluoropyrimidines are required to optimize dosing. Such trials need to take into account the well-known sex differences in their effects, as well as other parameters, such as individual body composition determined by CT scan, DPD phenotype and/or mutations, and pharmacokinetics. 


\section{Funding}

This work was supported by the National Cancer Institute [grants number U10CA180882 (NCCTG/Alliance); 180822 and 180868 (NRG Oncology)].

\section{Notes}

Role of the funder: The funding source had no role in the design of the study, the collection, analysis, and interpretation of the data, the writing of the manuscript and the decision to submit the manuscript for publication.

Disclaimers: The content is solely the responsibility of the authors and does not necessarily represent the official views of the National Institutes of Health.

Disclosures: AD Wagner has received travel support from Ipsen, Abbvie, Sanofi to my institution and consultation fees: BMS, Merck, Servier, MSD, Bayer, Lilly, Celgene, Shire, Pfizer to my institution. I am coordinating investigator of EORTC trial 1203, supported by an educational grant from Roche to EORTC, all outside the submitted work. TA has served in a consulting/advisory role and or received honoraria for, Amgen, Bristol-Myers Squibb, Chugai, Clovis, Halliodx, MSD Oncology, Pierre Fabre, Roche/Ventana, Sanofi, Servier and has received travel, accommodations, and expenses from Roche/Ventana, MSD Oncology, and Bristol-Myers Squibb outside the submitted work. E Francini has received travel and accommodation support from Janssen-Cilag and grant support from Roche. J Taieb has received honoraria for speaker or advisory role from Merck, Roche, Amgen, Eli Lilly, Sanofi, MSD, Servier, Pierre-Fabre, Sirtex. T George has received research funding from BMS, Merck, Astra-Zeneca/Medimmune, Lilly, 
Bayer, Incyte, Tesaro, Pharmacyclics, Ipsen, Seattle Genetics, Newling Genetics. R Goldberg has received honoraria from Amgen, a consulting or advisory role for Merck, Taiho Pharmaceutical, Merck KGaA, and Novartis and received travel/accommodation expenses from Meck KG aA, Merck, and Amgen. Q Shi has stock and other ownership interests for Amgen (herself) Johnson and Johson (herself), an advisory role for Yiviva Inc. (herself), and research funding from Celgene (to her institution) and Roche/Genentech (to her institution). All remaining authors have declared no conflict of interest.

Prior presentations: This study has been presented as poster at the American Society for Clinical Oncology Annual Meeting 2018 (abstract 3606).

Data availability: The data sharing of individual patient data from each participating trial will be subject to the policy and procedures of the institutions and groups who conducted the original study.

\section{References}

1. Legato MJ, Johnson PA, Manson JE. Consideration of Sex Differences in Medicine to Improve Health Care and Patient Outcomes. JAMA 2016;316(18):1865-1866.

2. Tannenbaum C, Ellis RP, Eyssel F, et al. Sex and gender analysis improves science and engineering. Nature 2019;575(7781):137-146. 
3. Docherty JR, Stanford SC, Panattieri RA, et al. Sex: A change in our guidelines to authors to ensure that this is no longer an ignored experimental variable. Br J Pharmacol 2019;

10.1111/bph.14761.

4. Schiebinger L, Leopold SS, Miller VM. Editorial policies for sex and gender analysis. The Lancet 2016;388(10062):2841-2842.

5. The editor perspective: implementing journal editorial policies. In: Theresa M Wizemann Sex-Specific Reporting of Scientific Research: A Workshop Summary. The National Academies Press, Washington (DC); 2012.

6. Wagner AD, Oertelt-Prigione S, Adjei A, et al. Gender medicine and oncology: report and consensus of an ESMO workshop. Ann Oncol 2019;30(12):1914-1924.

7. Reisner SI, Poteat T, Keatley J, et al. Global Health Burden and Needs of Transgender Populations: A Review. Lancet 2016;388(10042):412-436.

8. Cheung WY, Shi Q, O'Connell M, et al. The predictive and prognostic value of sex in early-stage colon cancer: a pooled analysis of 33,345 patients from the ACCENT database. Clin Colorectal Cancer 2013;12(3):179-87.

9. Cheung WY, Renfro LA, Kerr D, et al. Determinants of Early Mortality Among 37,568 Patients With Colon Cancer Who Participated in 25 Clinical Trials From the Adjuvant Colon Cancer Endpoints Database. J Clin Oncol 2016;34(11):1182-9.

10. Soldin OP, Mattison DR. Sex differences in pharmacokinetics and pharmacodynamics. Clin Pharmacokinet 2009;48(3):143-57. 
11. Nikanjam $\mathrm{M}$, Stewart $\mathrm{CF}$, Takimoto $\mathrm{CH}$, et al. Population pharmacokinetic analysis of oxaliplatin in adults and children identifies important covariates for dosing. Cancer Chemother Pharmacol 2015;75(3):495-503.

12. Port RE, Daniel B, Ding RW, et al. Relative importance of dose, body surface area, sex, and age for 5-fluorouracil clearance. Oncology 1991;48(4):277-81.

13. Mueller F, Buchel B, Koberle D, et al. Gender-specific elimination of continuousinfusional 5-fluorouracil in patients with gastrointestinal malignancies: results from a prospective population pharmacokinetic study. Cancer Chemother Pharmacol 2013;71(2):36170.

14. Milano G, Etienne MC, Cassuto-Viguier E, et al. Influence of sex and age on fluorouracil clearance. J Clin Oncol 1992;10(7):1171-5.

15. Milano G, Etienne MC, Pierrefite V, et al. Dihydropyrimidine dehydrogenase deficiency and fluorouracil-related toxicity. Br J Cancer 1999;79(3-4):627-30.

16. Etienne MC, Lagrange JL, Dassonville O, et al. Population study of dihydropyrimidine dehydrogenase in cancer patients. J Clin Oncol 1994;12(11):2248-53.

17. Lu Z, Zhang R, Diasio RB. Dihydropyrimidine dehydrogenase activity in human peripheral blood mononuclear cells and liver: population characteristics, newly identified deficient patients, and clinical implication in 5-fluorouracil chemotherapy. Cancer Res 1993;53(22):54338.

18. Schwab M, Zanger UM, Marx C, et al. Role of genetic and nongenetic factors for fluorouracil treatment-related severe toxicity: a prospective clinical trial by the German 5-FU Toxicity Study Group. J Clin Oncol 2008;26(13):2131-8. 
19. Lee AM, Shi Q, Pavey E, et al. DPYD variants as predictors of 5-fluorouracil toxicity in adjuvant colon cancer treatment (NCCTG N0147). J Natl Cancer Inst 2014;106(12).

20. Meulendijks D, Henricks LM, Sonke GS, et al. Clinical relevance of DPYD variants c.1679T>G, c.1236G >A/HapB3, and c.1601G >A as predictors of severe fluoropyrimidineassociated toxicity: a systematic review and meta-analysis of individual patient data. Lancet Oncol 2015;16(16):1639-50.

21. Gusella M, Toso S, Ferrazzi E, et al. Relationships between body composition parameters and fluorouracil pharmacokinetics. Br J Clin Pharmacol 2002;54(2):131-9.

22. Wagner AD, Rakez $M$, Chibaudel, $B$ et al. Sex differences in efficacy and toxicity of first-line treatment of metastatic colorectal cancer (CRC) : An analysis of 18.399 patients in the ARCAD database. Poster presented at the 2020 Virtual meeting of the American Society for Clinical Oncology; J Clin Oncol 28, no. 15_suppl (May 20, 2020) 4029-4029.

23. Gurney H. How to calculate the dose of chemotherapy. Br J Cancer 2002;86(8):1297302.

24. Newell DR. Getting the right dose in cancer chemotherapy--time to stop using surface area? Br J Cancer 2002;86(8):1207-8.

25. Innominato PF, Giacchetti S, Moreau T, et al. Prediction of survival by neutropenia according to delivery schedule of oxaliplatin-5-Fluorouracil-leucovorin for metastatic colorectal cancer in a randomized international trial (EORTC 05963). Chronobiol Int 2011;28(7):586-600.

26. Yoshino T, Cleary JM, Van Cutsem E, et al. Neutropenia and survival outcomes in metastatic colorectal cancer patients treated with trifluridine/tipiracil in the RECOURSE and J003 trials. Ann Oncol 2020;31(1):88-95. 
27. Gamelin E, Boisdron-Celle M, Delva R, et al. Long-term weekly treatment of colorectal metastatic cancer with fluorouracil and leucovorin: results of a multicentric prospective trial of fluorouracil dosage optimization by pharmacokinetic monitoring in 152 patients. J Clin Oncol $1998 ; 16(4): 1470-8$.

28. Beumer JH, Chu E, Allegra C, et al. Therapeutic Drug Monitoring in Oncology: International Association of Therapeutic Drug Monitoring and Clinical Toxicology Recommendations for 5-Fluorouracil Therapy. Clin Pharmacol Ther 2018; 10.1002/cpt.1124. 29. Cristina V, Mahachie J, Mauer M, et al. Association of Patient Sex With ChemotherapyRelated Toxic Effects: A Retrospective Analysis of the PETACC-3 Trial Conducted by the EORTC Gastrointestinal Group. JAMA Oncol 2018;4(7):1003-1006.

30. Gamelin E, Delva R, Jaob J, et al. Individual Fluouracil Dose Adjustment Based on Pharmacokinetic Follow-Up Compared With Conventional Dosage: Results of a Multicenter Randomized Trial of Patients With Metastatic Colorectal Cancer. J Clin Oncol 2008;26(13):2099105. 


\section{TABLES}

Table 1. Patient Characteristics by Chemotherapy Regimen

\begin{tabular}{|c|c|c|c|c|}
\hline Characteristic & Female & Male & Total & $P$-value ${ }^{1}$ \\
\hline All patients, No. & 15976 & 18664 & 34640 & \\
\hline Age, y No. (\%) & & & & $<.001$ \\
\hline$<50$ & $3022(18.9)$ & $3142(16.8)$ & $6164(17.8)$ & \\
\hline $50-64$ & $7153(44.8)$ & $8519(45.6)$ & $15672(45.2)$ & \\
\hline$\geq 65$ & $5801(36.3)$ & $7002(37.5)$ & $12803(37.0)$ & \\
\hline Missing & 0 & 1 & 1 & \\
\hline BMI, kg/m² No. (\%) & & & & $<.001$ \\
\hline$<18.5$ & $645(4.5)$ & $208(1.2)$ & $853(2.8)$ & \\
\hline $18.5-25$ & $6631(46.3)$ & $6573(39.4)$ & $13204(42.6)$ & \\
\hline$\geq 25$ & 7056 (49.\%) & 9889 (59.3) & $16945(54.7)$ & \\
\hline Missing & 1644 & 1994 & 3638 & \\
\hline Performance Score, n (\%) & & & & $<.001$ \\
\hline 0 & $12224(78.5)$ & $14675(80.5)$ & $26899(79.6)$ & \\
\hline 1 & $3233(20.8)$ & $3383(18.6)$ & $6616(19.6)$ & \\
\hline 2 & $116(0.7)$ & $158(0.9)$ & $274(0.8)$ & \\
\hline 3 & $2(0.0)$ & $4(0.0)$ & $6(0.0)$ & \\
\hline Missing & 401 & 444 & 845 & \\
\hline 5FU Patients & 10521 & 12259 & 22780 & \\
\hline Age, y No. (\%) & & & & $<.001$ \\
\hline$<50$ & $1958(18.6)$ & $1990(16.2)$ & $3948(17.3)$ & \\
\hline $50-64$ & $4613(43.8)$ & 5501 (44.9) & $10114(44.4)$ & \\
\hline$\geq 65$ & 3950 (37.5) & 4768 (38.9) & 8718 (38.3) & \\
\hline BMI, kg/m² No. (\%) & & & & $<.001$ \\
\hline$<18.5$ & $412(4.6)$ & $138(1.3)$ & $550(2.9)$ & \\
\hline $18.5-25$ & $4187(47.1)$ & 4100 (39.9) & 8287 (43.2) & \\
\hline$\geq 25$ & $4300(48.3)$ & $6043(58.8)$ & $10343(53.9)$ & \\
\hline Missing & 1622 & 1978 & 3600 & \\
\hline Performance Score, No. (\%) & & & & $<.001$ \\
\hline 0 & 7851 (77.2) & $9408(79.4)$ & $17259(78.4)$ & \\
\hline 1 & $2203(21.7)$ & $2292(19.3)$ & $4495(20.4)$ & \\
\hline 2 & $109(1.1)$ & $149(1.3)$ & $258(1.2)$ & \\
\hline 3 & $1(0.0)$ & $4(0.0)$ & $5(0.0)$ & \\
\hline Missing & 357 & 406 & 763 & \\
\hline FOLFOX Patients & 2720 & 3149 & 5869 & \\
\hline Age, y No. (\%) & & & & .08 \\
\hline$<50$ & $573(21.1)$ & $608(19.3)$ & $1181(20.1)$ & \\
\hline $50-64$ & $1315(48.3)$ & $1502(47.7)$ & $2817(48.0)$ & \\
\hline$\geq 65$ & $832(30.6)$ & $1039(33.0)$ & $1871(31.9)$ & \\
\hline BMI, kg/m² No. (\%) & & & & $<.001$ \\
\hline$<18.5$ & $106(3.9)$ & $20(0.6)$ & $126(2.1)$ & \\
\hline $18.5-25$ & $1183(43.5)$ & $1134(36.0)$ & 2317 (39.5) & \\
\hline$\geq 25$ & $1430(52.6)$ & 1995 (63.4) & $3425(58.4)$ & \\
\hline Missing & 1 & 0 & 1 & \\
\hline Performance Score, No. (\%) & & & & .53 \\
\hline 0 & 2247 (83.2) & $2621(83.9)$ & $4868(83.6)$ & \\
\hline 1 & $449(16.6)$ & 495 (15.9) & $944(16.2)$ & \\
\hline 2 & $4(0.1)$ & $7(0.2)$ & $11(0.2)$ & \\
\hline 3 & $1(0.0)$ & $0(0.0)$ & $1(0.0)$ & \\
\hline
\end{tabular}




\begin{tabular}{|c|c|c|c|c|}
\hline Missing & 19 & 26 & 45 & \\
\hline Capecitabine Patients & 888 & 1026 & 1914 & \\
\hline Age, y, No. (\%) & & & & .17 \\
\hline$<50$ & $121(13.6)$ & $141(13.8)$ & $262(13.7)$ & \\
\hline $50-64$ & $387(43.6)$ & $487(47.5)$ & $874(45.7)$ & \\
\hline$\geq 65$ & $380(42.8)$ & $397(38.7)$ & $777(40.6)$ & \\
\hline Missing & 0 & 1 & 1 & \\
\hline BMI, kg/m² No.(\%) & & & & $<.001$ \\
\hline$<18.5$ & $42(4.8)$ & $13(1.3)$ & $55(2.9)$ & \\
\hline $18.5-25$ & $434(49.2)$ & $449(44.2)$ & $883(46.5)$ & \\
\hline$\geq 25$ & $406(46.0)$ & $554(54.5)$ & $960(50.6)$ & \\
\hline Missing & 6 & 10 & 16 & \\
\hline Performance Score, No. (\%) & & & & 84 \\
\hline 0 & $750(84.8)$ & $867(84.5)$ & $1617(84.7)$ & \\
\hline 1 & $134(15.2)$ & $159(15.5)$ & $293(15.3)$ & \\
\hline Missing & 4 & 0 & 4 & \\
\hline CAPOX Patients & 850 & 1014 & 1864 & \\
\hline Age, y No. (\%) & & & & 10 \\
\hline$<50$ & $146(17.2)$ & $176(17.4)$ & $322(17.3)$ & \\
\hline $50-64$ & $361(42.5)$ & $475(46.8)$ & $836(44.8)$ & \\
\hline$\geq 65$ & $343(40.4)$ & $363(35.8)$ & 706 (37.9) & \\
\hline BMI, kg/m² No. (\%) & & & & $<.001$ \\
\hline$<18.5$ & $43(5.1)$ & $20(2.0)$ & $63(3.4)$ & \\
\hline $18.5-25$ & $376(44.2)$ & $415(40.9)$ & $791(42.4)$ & \\
\hline$\geq 25$ & $431(50.7)$ & $579(57.1)$ & $1010(54.2)$ & \\
\hline Performance Score, No. (\%) & & & & .03 \\
\hline 0 & 607 (72.3) & $774(76.8)$ & $1381(74.7)$ & \\
\hline 1 & $233(27.7)$ & $234(23.2)$ & $467(25.3)$ & \\
\hline Missing & 10 & 6 & 16 & \\
\hline FOLFIRI Patients & 997 & 1216 & 2213 & \\
\hline Age, y No. (\%) & & & & .005 \\
\hline$<50$ & $224(22.5)$ & $227(18.7)$ & $451(20.4)$ & \\
\hline $50-64$ & $477(47.8)$ & $554(45.6)$ & $1031(46.6)$ & \\
\hline$\geq 65$ & $296(29.7)$ & $435(35.8)$ & $731(33.0)$ & \\
\hline BMI, $\mathrm{kg} / \mathrm{m}^{2}$ No. (\%) & & & & $<.001$ \\
\hline$<18.5$ & $42(4.3)$ & $17(1.4)$ & $59(2.7)$ & \\
\hline $18.5-25$ & $451(45.9)$ & $475(39.3)$ & $926(42.2)$ & \\
\hline$\geq 25$ & $489(49.8)$ & $718(59.3)$ & $1207(55.1)$ & \\
\hline Missing & 15 & 6 & 21 & \\
\hline Performance Score, No. (\%) & & & & .01 \\
\hline 0 & 769 (78.0) & $1005(83.1)$ & $1774(80.8)$ & \\
\hline 1 & $214(21.7)$ & $203(16.8)$ & $417(19.0)$ & \\
\hline 2 & $3(0.3)$ & $2(0.2)$ & $5(0.2)$ & \\
\hline Missing & 11 & 6 & 17 & \\
\hline
\end{tabular}

${ }^{1}$ Chi-Square $\mathrm{p}$-value for differences between male and female patients, statistical significance level <.001. 


\section{Figure Titles and Legends}

Figure 1. Adjusted Odds Ratios (95\% confidence intervals) for grade III/IV hematological toxicities (log base 10 scale). *Stratified by study and treatment arm, adjusted for age, stage grade, PS, and $\mathrm{BMI} ; * *$ Stratified Wald p-value; 5FU=Fluorouracil therapy, FOLFOX=Leucovorin + Fluorouracil + Oxaliplatin therapy, CAPOX=Capecitabine + Oxaliplatin therapy, $\mathrm{Cl}=\mathrm{Confidence}$ Interval, PS=Performance Status, BMI=Body Mass Index

Figure 2. Adjusted Odds ratios (95\% confidence intervals) for grade III/IV non-hematological toxicities (log base 10 scale). *Stratified by study and treatment arm, adjusted for age, stage grade, PS, and $\mathrm{BMI} ; * *$ Stratified Wald p-value; 5FU=Fluorouracil therapy, FOLFOX=Leucovorin + Fluorouracil + Oxaliplatin therapy, CAPOX=Capecitabine + Oxaliplatin therapy, $\mathrm{Cl}=\mathrm{Confidence}$ Interval, PS=Performance Status, BMI=Body Mass Index 


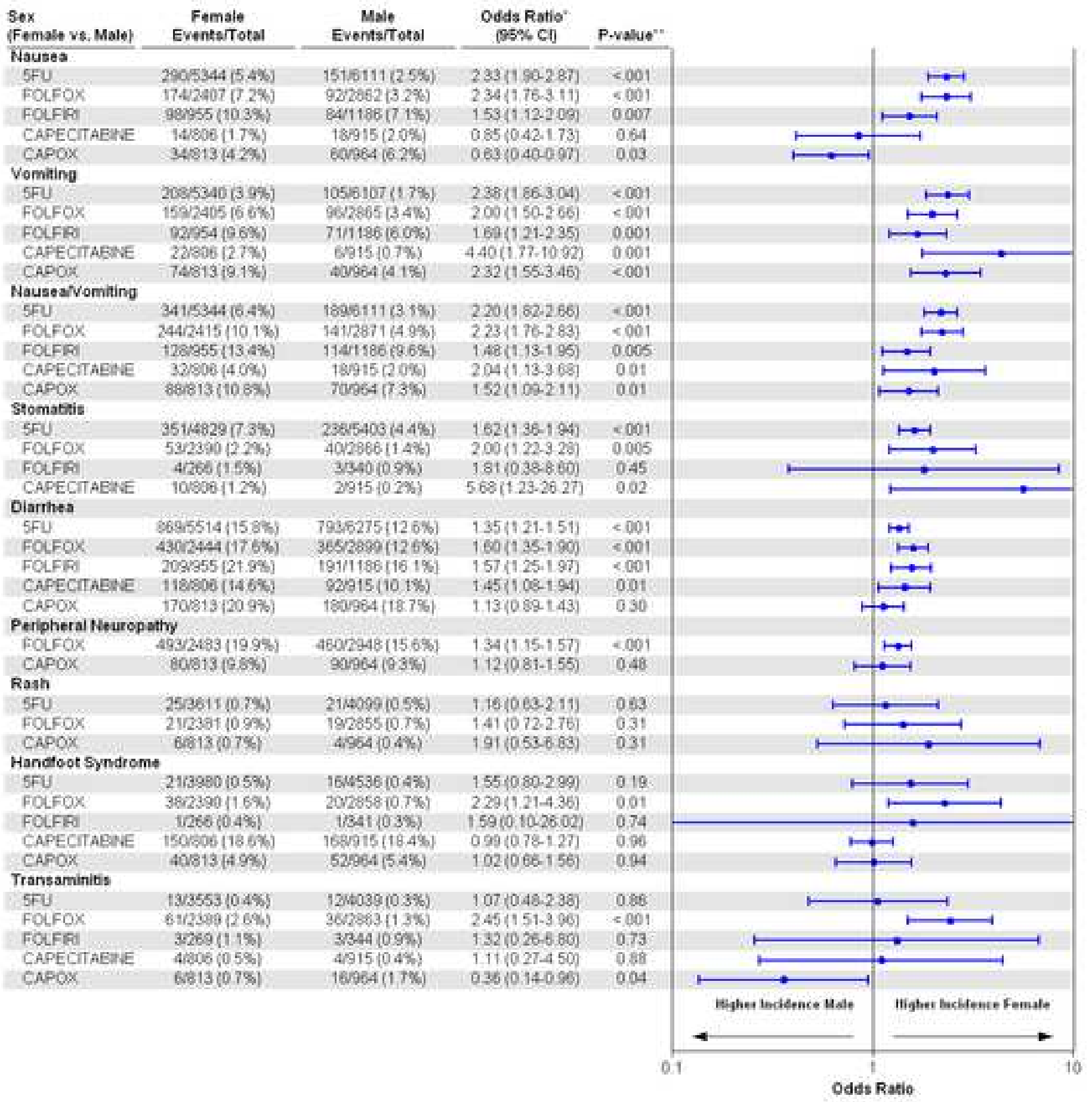




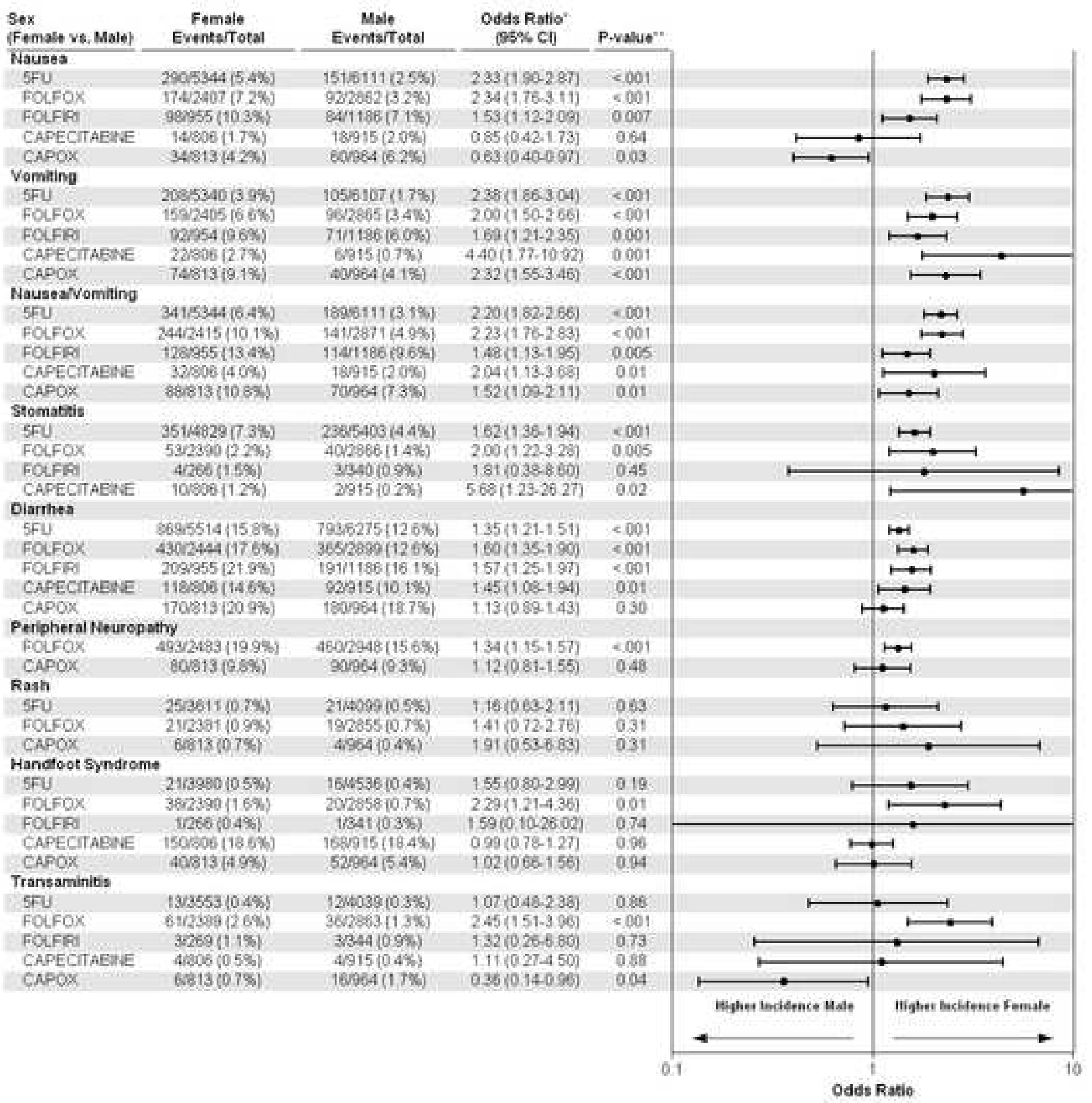


